\title{
Title
}

Contemporary Women's Secure Psychiatric Services in the United Kingdom: A

\section{Qualitative Analysis of Staff Views}

\section{Authors}

Dr Tammi Walker, Dr Dawn Edge, Professor Jenny Shaw, Dr Hannah Wilson, Dr Louisa McNair, Heather Mitchell, Dr Kerry Gutridge, Dr Jane Senior, Professor Matthew Sutton, Rachel Meacock, and Professor Kathryn Abel

\section{Dr Tammi Walker, PhD (corresponding author)}

University of Manchester, Division of Psychology and Mental Health, School of Health Sciences, Faculty of Biology, Medicine and Health, Room 2.310 Jean McFarlane Building, Manchester, M13 9PL.

Tammi.walker@manchester.ac.uk

01613758087

\section{Dr Dawn Edge, PhD}

University of Manchester, Division of Psychology and Mental Health, School of Health Sciences, Faculty of Biology, Medicine and Health, Jean McFarlane Building, Manchester, M13 9PL

Dawn.edge@manchester.ac.uk

\section{Professor Jenny Shaw, PhD, MB ChB}

Offender Health Research Network, University of Manchester, Division of Psychology and Mental Health, School of Health Sciences, Faculty of Biology, Medicine and Health, Jean McFarlane Building, Manchester, M13 9PL.

jennifer.j.shaw@manchester.ac.uk

\section{Dr Hannah Wilson, DClinPsy}

Lancashire Care NHS Foundation Trust, Preston, United Kingdom. Hannah.wilson@lancashirecare.nhs.uk

This is the author manuscript accepted for publication and has undergone full peer review but has not been through the copyediting, typesetting, pagination and proofreading process, which may lead to differences between this version and the Version of Record. Please cite this article as doi: $10.1111 /$ ipm.12416

This article is protected by copyright. All rights reserved 


\section{Dr Louisa McNair, DClinPsy}

Cheshire and Wirral Partnership NHS Foundation Trust, Chester, United Kingdom. Louisa.mcnair@cwp.nhs.uk

\section{Heather Mitchell, BA}

Centre for Women's Mental Health, Division of Psychology and Mental Health, School of Health Sciences, Faculty of Biology, Medicine and Health, University of Manchester, Jean McFarlane Building, Manchester, M13 9PL

heather.mitchell@manchester.ac.uk

\section{Dr Kerry Gutridge, PhD}

Centre for Women's Mental Health, Division of Psychology and Mental Health, School of Health Sciences, Faculty of Biology, Medicine and Health, University of Manchester, Jean McFarlane Building, Manchester, M13 9PL

Kerry.gutridge@manchester.ac.uk

\section{Dr Jane Senior, PhD}

Offender Health Research Network, University of Manchester, Division of Psychology and Mental Health, School of Health Sciences, Faculty of Biology, Medicine and Health, Jean McFarlane Building, Manchester, M13 9PL.

jane.senior@manchester.ac.uk

\section{Professor Matt Sutton, PhD}

University of Manchester, School of Health Sciences, Centre for Health Economics, Jean McFarlane Building, Manchester, M13 9PL.

matt.sutton@manchester.ac.uk

\section{Rachel Meacock, MA}

University of Manchester, School of Health Sciences, Centre for Health Economics, Jean McFarlane Building, Manchester, M13 9PL.

rachel.meacock@manchester.ac.uk

\section{Professor Kathryn Abel, PhD, MB ChB}


Centre for Women's Mental Health, Division of Psychology and Mental Health, School of Health Sciences, Faculty of Biology, Medicine and Health, University of Manchester, Jean McFarlane Building, Manchester, M13 9PL

Kathryn.abel@manchester.ac.uk

\section{Acknowledgments}

The authors would like to thank all staff who were interviewed as part of this research. We would like to thank Kathryn Harney and Annie Bartlett for their expert guidance and advice. We would also like to express our gratitude to Boo Wheatcroft and the Mental Health Research Network (MHRN) for their valuable contribution, and all staff who facilitated data collection.

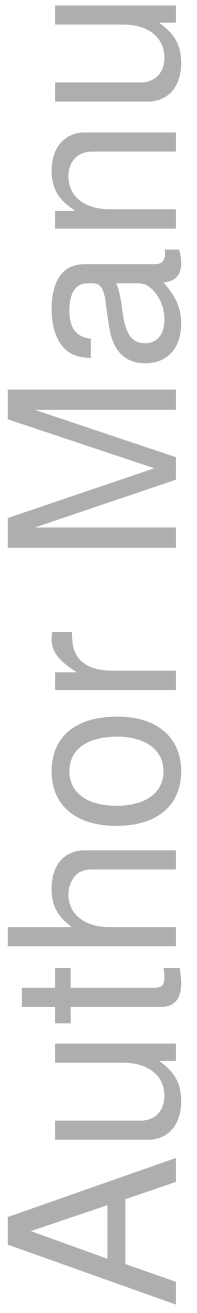




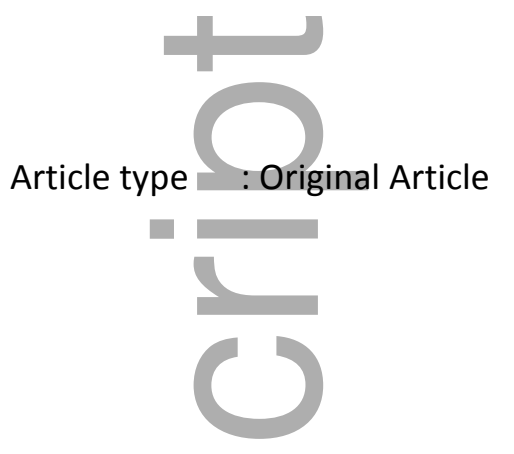

\section{Accessible summary}

\section{What is known on the subject?}

- Three pilot UK-only Women's Enhanced Medium Secure Services (WEMSS) opened in 2007 to support women's movement from high secure care and provide a bespoke, women-only service.

- Evidence suggests that women's secure services are particularly challenging environments to work in and staffing issues (e.g. high turnover) can cause difficulties in establishing a therapeutic environment.

- Research in this area has focused on the experiences of service users. Studies which have examined staff views have focused on their feelings towards women in their care and the emotional burden of working in women's secure services. No papers have made a direct comparison between staff working in different services.

\section{What does this paper add to existing knowledge?}

- This is the first study to explore the views and experiences of staff in the three UK WEMSS pilot services and contrast them with staff from women's medium secure services.

- Drawing upon data from eighteen semi-structured interviews (nine WEMSS; nine non-WEMSS), key themes cover staff perceptions of factors important for women's recovery and their views on operational aspects of services.

- This paper extends our understanding of the experiences of staff working with women in secure care and bears relevance for staff working internationally, as well as in UK services. 


\section{What are the implications for practice?}

- The study reveals the importance of induction and training for bank and agency staff working in women's secure services.

- Further, regular clinical supervision should be mandatory for all staff so they are adequately supported.

$\square$

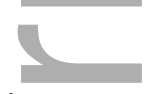

\section{Abstract}

\section{Introduction}

Women's Enhanced Medium Secure Services (WEMSS) are bespoke, gender-sensitive services which opened in the UK in 2007 at three pilot sites. This study is the first of its kind to explore the experiences of WEMSS staff, directly comparing them to staff in a standard medium secure service for women. The literature to date has focused on the experiences of service users, or staff views on working with women in secure care.

\section{Aim}

This qualitative study, embedded in a multi-method evaluation of WEMSS, aimed to explore the views and experiences of staff in WEMSS and comparator medium secure services.

\section{Methods}

Qualitative interviews took place with nine WEMSS staff and nine comparator medium secure staff. Interviews focused on factors important for recovery, barriers to facilitating recovery and operational aspects of the service.

\section{Discussion}

This study provides a rare insight into the perspectives of staff working in UK women's secure services, an under-researched area in the UK and internationally. Findings suggest that the success of services, including WEMSS, is compromised by operational factors such as the use of bank staff.

\section{Implications for Practice}

Comprehensive training and supervision should be mandatory for all staff, so best practice is met and staff adequately supported. 


\section{Relevance Statement}

The current paper explores the views of staff employed in UK Women's Enhanced Medium Secure Services (WEMSS) compared to staff working in non-WEMSS. Semi-structured interviews explored staff perceptions of what is important for women's recovery and of operational aspects of their service. The study reveals the importance of staff induction and training for bank and agency staff working with complex women in such secure services. Further, regular clinical supervision should be made mandatory to all staff so they are adequately supported and can engage in best practice.

\section{Introduction}

In the United Kingdom the past 20 years have seen significant changes in women's secure mental health services, driven by concerns about women's vulnerability in shared-sex accommodation (Howlett, 1994; Mezey, Hassell, \& Bartlett, 2005; Shaw, Davies, \& Morey, 2001) and recognition that women were being inappropriately detained in high security psychiatric hospitals because of complex mental health problems rather than risk to the public (Aiyegbusi \& Kelly, 2012). Consequently, a new and innovative type of gender-sensitive medium secure service known as Women's Enhanced Medium Secure Services (WEMSS) was conceived (Department of Health, 2002, 2003).

WEMSS services were piloted at three UK sites, aiming to facilitate closure of women's high secure beds at Ashworth and Broadmoor and to provide a bespoke, properly resourced, 'Women only' service (Edge, 2005). WEMSS set out to provide enhanced relational security, an organic process informed by attachment theory and based on staff members' knowledge and understanding of a woman and the environment (ComptonDickinson, Odell-Miller, \& Adlam, 2012).

An evaluation strategy was developed to determine whether WEMSS should become an established model of care (Edge, 2005). This included a case control study comparing the clinical, functional and social outcomes of women in WEMSS to those of matched control women in non-WEMSS facilities. This study found that WEMSS did not achieve better outcomes for women in terms of risk and clinical characteristics and transition to lower security levels (Edge et al., 2016). Service users in both types of service described good relationships with staff, but some women suggested that low staff numbers and bank staff could interfere with building trusting, therapeutic relationships (Abel et al., 2011). 
The current study focuses on interviews with WEMSS and non-WEMSS practitioners and to our knowledge is the first of its kind. A search of the international literature revealed only a handful of studies that have explored the views of staff working in women's secure services and the majority of these are UK-based (Baker \& McKay, 2001; Nathan et al 2007; Bartlett \& Somers, 2016; Beryl, Davies, \& Völlm, 2016; Kurtz \& Jeffcote, 2011). The only non-UK article revealed by the search was a pilot study which used the Feelings Word Checklist to examine staff emotional responses to women in their care (De Vogel \& Louppen, 2016). UK-based research has focused on the emotional burdens placed on staff, and the impact of the environment on staff working with women in secure environments (Nathan et al 2007; Kurtz et al, 2011; Beryl, Davies, \& Völlm, 2016). A small number of research papers have examined staff perceptions of service user experiences (Baker \& McKay, 2001) and operational issues (Bartlett \& Somers, 2016) but these have not directly compared delivery in two distinct types of service.

Given that practitioners working in forensic services are at risk of occupational stress and burnout (e.g., Elliott \& Daley, 2013), it is crucial that their views are heard to ensure they are supported in their roles. Further, since good interpersonal relationships are a central aspect of caring for women (Archer, Lau, \& Sethi, 2016) and of WEMSS (Edge, 2005), it is important to explore staff perceptions of women's needs and how well they are being met.

Qualitative interviews with staff in high secure women's services highlight the challenging situations that staff face daily e.g. fear about responding to incidences of selfharm. Staff conflicts were also regarded as one of the most challenging situations, emphasising the importance of team work and being surrounded by the right people (Beryl, Davies, \& Völlm, 2016). However, staffing issues are frequently reported in women's services (Bartlett \& Somers, 2016; Parry-Crooke \& Stafford, 2009). During the national evaluation of women's secure care pathways in 2011 and 2012, senior practitioners raised concerns about sick leave, burnout and inconsistent staffing (Bartlett \& Somers, 2016).

Department of Health Guidelines 'Mainstreaming gender and women's mental health: implementation guidance', advocate that staff support includes access to supervision and a space for reflective practice (Department of Health, 2003). Clinical supervision is viewed as an essential way of supporting staff in difficult working environments including secure services caring for women with personal disorders (Logan \& Taylor, 2017), yet uptake is low in women's secure services (Long, Harding, Payne \& Collins, 2014). Parry-Crooke and Stafford (2009) suggested that women's secure services require increased resources and monitoring by managers to ensure availability and uptake of supervision. The current study 
sought to elicit staff views on organisational aspects of their services including supervision and staff training. Such information was required to provide a thorough evaluation of WEMSS and to identify barriers to optimal service delivery.

From a staff perspective, stakeholder-agreed success criteria for WEMSS were that staff feel valued and supported, staff want to work in WEMSS, staff comment favourably on ethos and cultures of WEMSS and there is evidence that staff interventions are effective. The first three of these criteria were explored using staff surveys and interviews (Edge, 2005).

\section{Study Aims}

1) Explore the success of WEMSS from staff perspectives, focusing on staff perceptions of service users' experiences and service operation.

2) Explore staff perceptions of staffing arrangements, including staff levels and roles, training, supervision and personal development opportunities.

3) Compare the views of practitioners in WEMSS to those of practitioners in comparator nonWEMSS medium secure services.

\section{Methods}

\section{Ethics Approval}

Ethical approval was granted by County Durham and Tess Valley NHS Research Ethics Committee (10/H0905/13, $1^{\text {st }}$ June 2010). All services included provided individual, site-specific governance approval. The field work took place over a period of six months (December 2010 to May 2011) at the three WEMSS pilot sites and non-WEMSS medium secure sites.

\section{Research Team and Reflexivity}

Interviews were conducted by two female research assistants from the University of Manchester, Hannah Wilson and Louisa McNair. Both researchers were trained in qualitative methods by an experienced researcher. The researchers had no prior relationship with the research participants and participants were not aware of the researchers' personal goals or reasons for doing the research. Both researchers were interested in developing the skills necessary to work as a clinical psychologist. 


\section{Study Design}

\section{Theoretical framework}

This qualitative study was nested in a larger evaluation of WEMSS (Edge, 2005). The study used face-to-face interviews. The systematic method of thematic analysis was used; with this analytic strategy, data exploration and theory-construction are combined and theoretical developments are made in a 'bottom up' manner so as to be anchored to the data (Braun and Clarke, 2006).

\section{Participant Selection}

Purposive sampling (May \& Pope, 1995) was used to recruit participants, ensuring that the views of individuals with different roles (e.g., nurses, psychiatrists) were represented. Each service was provided with suggestions of which disciplines we wished to interview; the local collaborator provided appropriate individuals with the information sheet and they were given an opportunity to discuss the research and ask questions. Then, if staff wished to participate, informed consent was obtained and the interview took place.

In total, nine WEMSS and nine non-WEMSS practitioners were interviewed; service demands meant that the target of ten from each service was not achieved. No participants dropped out of the research.

\section{Setting}

Due to the restricted area over which the study occurred, for ethical reasons and to avoid potential identification of participants, we shall not disclose the specific settings involved (Clark, 2006). All staff were interviewed in a private room within their workplace. All staff were given a pseudonym and the gender of some participants was changed to prevent identification.

The roles of staff interviewed are summarised in Table 1.

\section{[INSERT TABLE 1]}

\section{Data Collection}

A review of the literature was conducted and informed the development of a semistructured interview schedule (see Appendix 1). Additional questions were adapted from the 
Parry-Crooke and Stafford (2009) interview. Amendments were made to the schedule after a pilot interview (not included in the main study data) and ethical review. The interview focused on staff views and experiences of providing care and treatment to service users. Staff were also prompted to discuss operational aspects of the service and barriers to delivering an optimal service. Interviews were audio-recorded, with participants' consent. No additional field notes were taken during the interviews. Due to time restrictions, transcripts were not returned to the participants for comment and correction.

\section{Analysis}

Transcripts were checked for accuracy and coded in NVivo by the two interviewers. A subset of the coded transcripts was then checked for accuracy by a $3^{\text {rd }}$ research team member. Due to time restrictions, participant checking of themes was not undertaken. Data were analysed using thematic analysis (Braun \& Clark, 2006). Themes were clustered together, noting overlaps and goodness of fit, to form categories which are reported here.

\section{Rigour}

There has been a great deal of debate about rigour in qualitative research (Grbich, 1999). Credibility or confidence in the data was gained by the research assistants' prolonged engagement with the data (Lincoln \& Guba, 1985). Consistency was maintained by keeping an audit trail and an independent researcher checking the decisions and analysis. Transferability (neutrality) was evaluated by providing the raw data to a colleague to allow them to interpret how themes emerged.

\section{Results}

The four main themes which emerged from both sets of staff interviews were 1) challenges of working with women in secure care 2) relational security 3) service user involvement and 4) factors important for women's recovery. In addition, staff comments about operational aspects of their service centred on three main topics; 1) staff recruitment and retention, 2) supervision and 3) training.

\section{Challenges of Working with Women in Secure Care}

WEMSS staff spoke about the challenges of managing complex interpersonal dynamics between women; the promotion of boundaries and trust between staff and the women was described as essential but sometimes a difficult balance to strike. 
Issues to do with attachment and trust and that kind of pushing boundaries and just gaining that trust can take a long, long time for our ladies because they are so kind of messed up with their previous histories. (Bill, WEMSS nursing staff)

Similarly, non-WEMSS staff voiced concerns about the dynamics of working with women:

I think everybody is striving really hard for them to get that support, frustrating is the fact that one day they will take it and another day they can't, so it's getting round those areas. (Harriet, non-WEMSS nursing staff)

Staff from both types of service described how complex interpersonal dynamics meant that women could impact both positively and negatively on each other.

What often has happened with the women is that, if somebody's on one-to-one, then they don't feel that they're getting the input that they need and, therefore, they will do something to get the one-to-one, or two-to-one; or actually, I'll try and push the boundaries a little bit more. It'd be like a domino effect really sort of with the women. (Molly, WEMSS managerial staff)

I think that patients at different stages of their treatment can help each other, and women are far better at doing that then men are. (Dave, non-WEMSS managerial staff)

WEMSS staff also perceived that sometimes women's challenging behaviour demonstrated attempts to test, dominate and/or cause splits within staff teams, thereby disrupting therapeutic alliances and potentially sabotaging recovery. Staff recognised this as a mechanism to test commitment to their care:

I think that's about them actually testing as well, you know, how much someone will work with them even though they're, or constantly trying to say go away, leave me alone... and that's their way of, I think, trying to say is this person going to work with me or are they just going to end up doing what's happened to me all my life. (Tom, WEMSS managerial staff) 
Some WEMSS staff reported being profoundly affected by the women's distress and finding the work challenging and often 'exhausting', whilst others found it particularly rewarding:

So I very much enjoy the challenges of that interpersonal, of those interpersonal dynamics, it is exhausting work though because it is continually challenging you but I guess that's what, in part, attracts me to it... (Jill, WEMSS psychological therapies staff)

\section{Relational Security}

Practitioners in both types of service discussed their understanding of relational security and its importance:

I think in the WEMSS service relational security is very important to the women and it's about attachment and it's about getting to know the women and it's about getting them to trust you and it's about allowing them the opportunity to actually ventilate their feelings ...it's about working with them to show them that they're now in a service that is able to manage their illness and able to manage their behaviours and are prepared to work with them no matter how long that takes and they won't be rejected. (Tom, WEMSS managerial staff)

Both WEMSS and non-WEMSS practitioners were generally positive about the benefits of relational security and outlined similar important factors for good relational security. For WEMSS staff this included positive communication between staff members and providing support for each other, multidisciplinary team working, appropriate staffing levels, consistency of approach and knowing/understanding women and their 'triggers'. Similarly, non-WEMSS staff felt that working as a multidisciplinary team, understanding each other's roles within the service and knowing/understanding the women were most important.

I would take away some of the structural security and replace it with relational security and create an environment where people were valued, staff and patients. Because if I don't value staff, why would I expect them to value the contribution of patients? (Dave, non-WEMSS managerial staff) 
Staff identified barriers to effective relational security; WEMSS staff highlighted an inconsistent therapeutic approach, poor staffing levels and use of bank/agency staff as the main threats:

What would make it more ideal if we could use regular staff all the time and not use bank and agency and that to me would make an ideal service...bank and agency staff don't really know the patients so, therefore, they're not able to identify if someone's behaving in a different way or someone's acting in a different way. (Tom, WEMSS managerial staff)

Similarly, non-WEMSS staff identified high staff turnover and using bank staff as most detrimental to relational security.

All the units have real staffing pressures, so all the units inevitably end up using bank staff and sometimes agency staff and when you start having large numbers of patients on observations you end up having large numbers of staff who aren't really with the unit just watching patients and it creates a very un-therapeutic atmosphere .... (Nigel, non-WEMSS consultant psychiatrist)

\section{Service User Involvement}

Several WEMSS staff felt their service encouraged women to have responsibility, choice and independence. Advocacy services were highlighted as being important for ensuring women had a 'voice'. However, staff also acknowledged that meaningful engagement could be difficult:

I think they are quite involved in terms of we will as a team have discussions with them about what we think is necessary and what we think is appropriate, and I suppose the ultimate choice for them is not to engage isn't it? It's so important to have a collaborative approach with them because I think it would just be so diminishing and disheartening to be just told this is how it's going to be, you know, that you can't even have a say in what tablets you take, for example. (Lisa, WEMSS consultant psychiatrist) 
Non-WEMSS staff agreed with the importance of involving women in their care and actively engaging them in treatment and, similar to WEMSS practitioners, highlighted some of the challenges of giving women choice and involvement when they are detained under the Mental Health Act (1983):

There's a limit to what you can offer them in choice, though, isn't there, but they're detained patients in a psychiatric hospital and they can't go anywhere unless the Ministry of Justice say otherwise. So you offer choice within that... (Catherine, non-WEMSS nursing staff)

\section{Factors Important for Women's Recovery}

Practitioners from WEMSS and non-WEMSS services identified similar factors as being important for recovery. For WEMSS staff, the most important factors focused on issues which have been previously outlined such as: positive relationships with staff, feeling valued, and having the ability to exercise choice.

WEMSS staff stated that the women needed to feel understood and to be actively involved in their care and recovery:

Being listened to, being involved in their care, not being put down but working on their skills, working on empowering them, working on their strengths and acknowledging that everybody does have difficult times but getting them to understand how to work through that... (Tom, WEMSS managerial staff)

Non-WEMSS staff highlighted similar factors that they felt were important in providing effective care to women, including having strong trusting relationships, encouraging service user involvement and acknowledging the complex needs of the client group.

Establishing trust with them. And respect. Honesty and openness... And I think in previous years, you know, up to recently I think probably a lot of services have not had that. And it's difficult to establish, but it's possible... I think we try extremely hard to establish that trust. And it's really difficult because not all staff buy into it... (Pam, non-WEMSS nursing staff) 


\section{Operational Successes or Barriers to Optimal Service Delivery}

Interviews also explored how staff felt about staffing arrangements and operational aspects of the service. Staff responses to these questions clustered under three main themes; recruitment and retention, staff supervision and staff training.

\section{Recruitment and Retention}

Staff often made a connection between recruitment and retention and staff commitment to working with women. A few WEMSS practitioners stated that they had an interest in women's mental health; their responses reflected their perception that they might belong to a self-selected group of staff who sought opportunities to work with complex women:

I've always been kind of interested in more the women's side. The staff that we've got at the moment were all very keen to work with women. It's a ward that most people wouldn't choose to work on unless they've got a particular interest, which is great for us because we want people who are interested in the ward to be on it. (Bill, WEMSS nursing staff)

Non-WEMSS practitioners also felt that staff should have a women-centred mind-set. They spoke of relishing the challenge of working with women:

I think at the minute you generally find that the majority of staff actually want to be here and work with the women which makes a big difference... it is a certain mind-set that works with these women and understands the philosophy of care. (Georgina, nonWEMSS nursing staff)

WEMSS staff suggested that managers now try to select staff on the basis of wanting to work with women:

When you're doing your recruitment and selection what we are now trying to do is focus on people who actually want to work with women because I think that's the only way that we can improve the service. (Tom, WEMSS managerial staff)

Non-WEMSS staff were more likely to report recruitment difficulties, particularly of qualified staff who wanted to work with women. There was also a suggestion that non- 
nursing roles might be at risk in financially- strained times. WEMSS' staff, however, reported greater stability among qualified, non-nursing staff.

Across the country services are being cut and services are looking at OTs [Occupational Therapists] being on the wards and on the numbers. And it's just a real concern. One, about the impact it's going to have on activity levels and us being able to do our job effectively. (Sam, non-WEMSS occupational therapy staff)

Non-WEMSS staff talked about negative attitudes to working with women and the associated stigma, which in their view remained current:

I think women's services are perceived as not as attractive as other services. I can look at the men's equivalent and they're fully staffed. Women are harder work, all you have to do is look at the level of events and injuries - incidents - they're a lot higher, and I suppose I can understand a member of staff wanting to go and work somewhere where they get paid the same for less hassle. (Catherine, non-WEMSS nursing staff)

\section{Staff Supervision}

Staff from both types of service reported receiving supervision and training. However, WEMSS staff reported more a formal supervision processes, with greater uptake and increased staff satisfaction:

We've also got a nurse consultant... she would actually be on the ward working with...primary nurses, working with the nursing staff in relation to how you manage maybe a difficult patient, how you manage a certain patient who has a high level of self-harm... looking at how the staff are doing and how to de-escalate, helping them formulate care plans around trigger factors, around relational security... (Tom, WEMSS managerial staff)

Non-WEMSS staff also talked about the importance of supervision and suggested the emphasis on the supervisory process was increasing: 
I think nursing in the past has been very much a poor relation, supervision, and I mean, most nurses will tell you that there's not enough supervision, and it just didn't exist on a formal basis. Always we've supported each other, always there's been a high level of support for staff in and out of work. But more recently training is becoming very much more important, starting like that. We now do clinical professional development, there's something every week. (Pam, non-WEMSS nursing staff)

Both WEMSS and non-WEMSS staff reported that formal supervision was not always taken up, due to time pressures or being perceived as ineffective. From non-WEMSS staff accounts, this was particularly apparent among nursing staff:

I think it's more of a challenge getting nursing staff to have clinical supervision. I think it always is. It's a lot easier to deliver within the other staff groups where you haven't got the same numbers of people; you haven't got night shifts and so forth. (Nigel, non-WEMSS consultant psychiatrist)

Staff from both types of service spoke about the importance of informal supervision and support. However, non-WEMSS staff perceived that informal support was not always available, particularly for new and unqualified staff:

I used to get an awful lot of support and I know how it all works, but for new people coming in, I think that is probably something that they find very hard because they're shocked. (Harriet, non-WEMSS nursing staff)

\section{Staff Training}

WEMSS staff generally expressed positive views about training opportunities, but suggested that the volume of mandatory training sometimes left little room for other forms of training:

I think the problem is we have, the trust is under such a lot of pressure for the mandatory training to be done that it's then very difficult to fit in all the other training.... (Jill, WEMSS psychological therapies staff)

Although most non-WEMSS staff felt that there was a good variety of training available, they felt it wasn't always adequately related to practice. They also highlighted a lack of training opportunities for unqualified staff: 
I think particularly for support workers there isn't the training that people need... There's no career path, basically, if you're not qualified. And I guess you can apply for courses, but they're not accredited, really. (Georgina, non-WEMSS nursing staff)

Non-WEMSS practitioners agreed that properly resourced training and development were necessary and important for service progression:

I do believe that we need to train people. I think if you're not growing your staff your organisation will shrivel and die. (Dave, non-WEMSS managerial staff)

\section{Discussion}

To our knowledge, this is the first study to explore and compare the views and experiences of staff in WEMSS and non-WEMSS services. Whilst WEMSS is a UK-based model of care, this study adds to the international evidence base by providing insight into the experiences of staff working with women in secure services. Until now, most qualitative research in this area has focused on the experiences of service users. Literature which has examined the views of staff has focused on their feelings towards women in their care and the emotional burden of working in secure services with women. The few papers which have examined staff perceptions of women's needs and operational issues have not made a direct comparison between different types of service. The current study found that staff from both services reported similar challenges of working with women and a clear commitment to involving women in their own care. In terms of service operation, both groups of staff reported similar concerns regarding staffing problems, supervision and training.

Relational security was a key feature and explicit aim of the UK WEMSS pilots; we found it was regarded as important across all services. Perceived barriers to successful implementation of relational security focused on poor staffing and the use of bank staff in both types of service. The Royal College of Psychiatrists 'Standards for medium secure units' states that the number of nursing staff must be sufficient to meet the needs of the patients at all times (Royal College of Psychiatrists, 2007); our findings indicate that these standards may not be being met across women's secure services. Absence of regular staff was perceived to damage relational security, consistent with previous findings that use of agency staff can shift the ward balance towards a more custodial, rather than therapeutic, environment (James, Fineberg, Shah \& Priest, 1990). In addition, non-WEMSS staff 
highlighted the lack of training opportunities for new or unqualified staff. Similar findings emerged in the 'My Life in Safe Hands' report, which found that unqualified staff were unlikely to receive supervision (Parry-Crooke \& Stafford, 2009). Earlier research has shown that mental health staff with less training are more critical and emotionally cold (Van Humbeeck \& Van Audenhove, 2003), highlighting the importance of effective training for bank or agency staff. In 2010, the Royal College of Psychiatry developed a training handbook on relational security for forensic services (Royal College of Psychiatrists, 2010) which explains the concept of relational security and how it should be implemented; we recommend that this is given to all staff. A recent paper on working with personality disordered women in secure care suggests that staff should be those who actively choose to work with women, and those with certain personal qualities including a good awareness of personal boundaries (Bodner et al., 2015; Logan \& Taylor, 2017). It is promising that staff in both WEMSS and non-WEMSS services demonstrated a passion for working with women, and that WEMSS selection processes was described as having a focus on recruiting staff who want to work with women.

Staff in both services raised concerns about staff uptake of supervision. Service users with personality disorders (a common diagnosis in secure services) have been shown to have substantial impact on carers and staff, including frequent exposure to behavioural 'acting out' of intolerable feelings, impulses and beliefs (Moore, 2012). It is concerning that, despite formal supervision procedures, poor uptake of supervision was an issue in WEMSS. Staff should be supported to attend supervision and receive training on the possible benefits, including enhancing creativity and reducing job-related strain (Berg \& Hallberg, 1999).

High service user involvement was described in both types of service, with staff in agreement that this would improve relational security, as well as empowering women. Staff accounts are consistent with those of service users; women in WEMSS spoke positively of being involved in decision-making about their treatment (Abel et al., 2011). However, recent research reported that across two large mental health trusts service users still feel marginalised in the care planning process (Grundy et al., 2015). If WEMSS have sustained the level of involvement in the pilot sites they should be commended for good practice in this area.

The authors acknowledge the delay in publishing this research. However, in the intervening period there has been little qualitative research into staff views and experiences (Beryl et al., 2016). This study provides valuable insight into operational challenges which need to be addressed in UK secure services and possibly further afield. A personal 
communication from NHS England informed us that WEMSS continue to provide 36 beds nationally. Since the WEMSS evaluation, approaches to care delivery have developed as the understanding of particular patient cohorts has matured. NHS England acknowledges that the need for secure pathways sensitive to the needs of women remains, and an updated commissioning strategy is currently underway to develop a gender-specific pathway (NHS England, 2016). We recommend that staff and service users are consulted throughout this developmental work to ensure that services are meeting the needs of key stakeholders.

\section{Strengths and Limitations}

This study has several strengths and is the first to directly compare the views of WEMSS and non-WEMSS practitioners. The qualitative design of this research used an inductive approach (Yin, 2011), allowing themes to be drawn from the data (Braun \& Clarke, 2006). The themes presented here are supported by evidence from the data itself (Braun \& Clarke, 2006; Guest, MacQueen, \& Namey, 2012). The techniques of thematic analysis (Braun \& Clarke, 2006) enabled the unique perceptions of individual participants to be recognised, which could have been rejected as anomalous using other methods (Braun \& Clarke, 2006; Flick, 2009).

A possible limitation is that the data collection took place from December 2010 to May 2011. However, we believe the findings still have clinical relevance as this is an underresearched area. It is important that staff views are heard and understood so that clinical practice continues to be informed accordingly (Walker, Logan, \& Shaw, 2017). Additionally, although purposive sampling was used, the small sample size must be acknowledged. Further, this is a UK-based study which limits the potential for findings to be generalised internationally.

\section{Implications for Practice}

The current study suggests that the success of women's secure services, including the WEMSS UK pilots, is compromised by operational factors which could put service users and staff at risk. Staff reported difficulties in the recruitment and retention of committed, regular staff which was seen as a potential threat to relational security. We recommend that regular training and supervision is available for all staff, to ensure that recommendations for best practice are being met and that staff are adequately supported and trained. Further, in line with staff views that all staff should have a women-centred mind-set, we would encourage women's services to emphasise this in selection procedures. 


\section{Funders}

This work was supported by the Department of Health; A clinical, economic and operational evaluation of the pilot Women's Enhanced Medium Secure Services (WEMSS). The views expressed in this paper do not represent those of the funders.

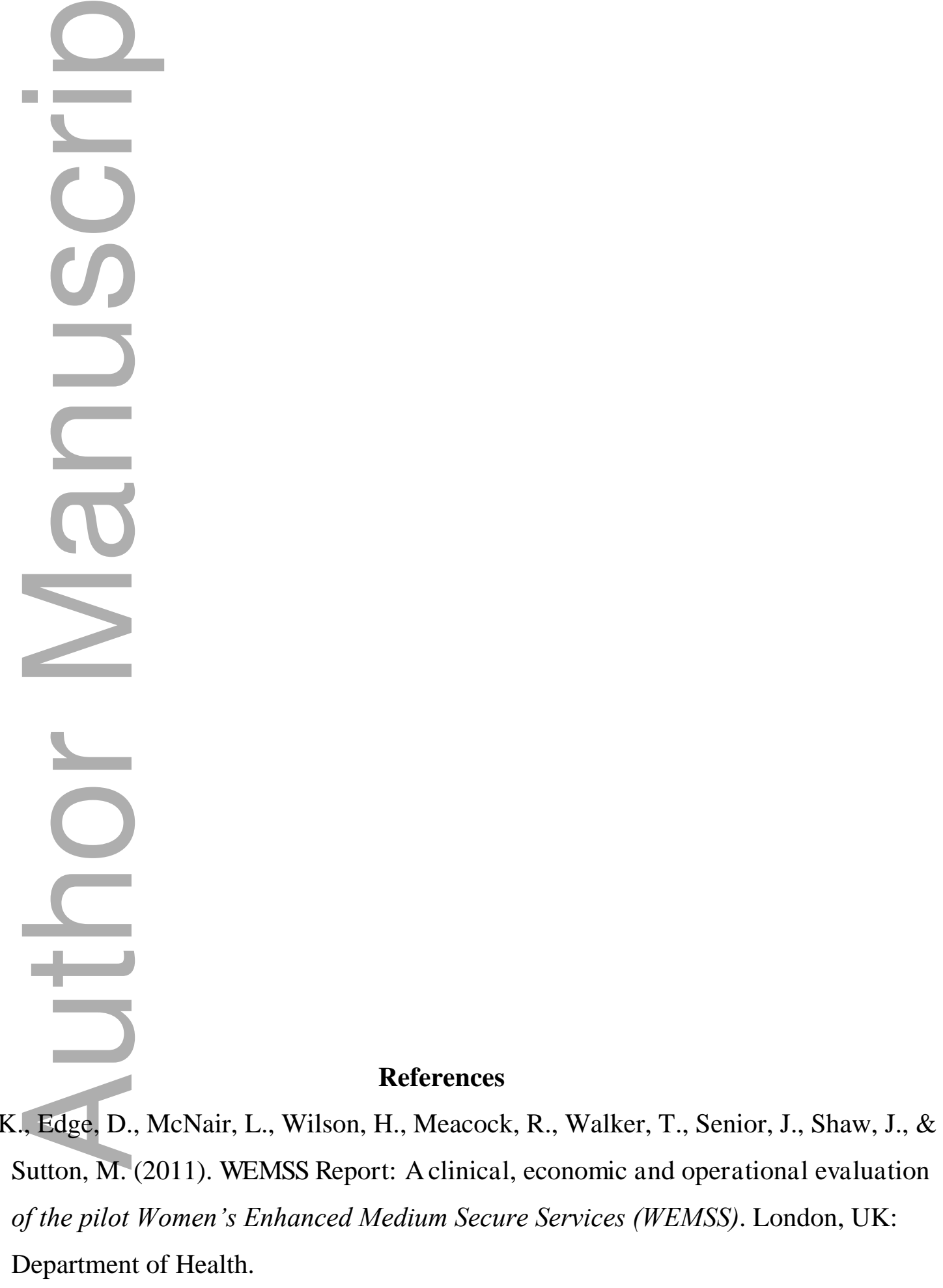


Aiyegbusi, A., \& Kelly, G. (Eds). (2012). Professional and therapeutic boundaries in forensic mental health practice. London, UK: Jessica Kingsley Publishers.

Archer, M., Lau, Y., \& Sethi, F. (2016). Women in acute psychiatric units, their characteristics and needs: A review. BJPsych Bulletin, 1-7. http://doi.org/10.1192/pb.bp.115.051573

Baker, S., \& McKay, E. A. (2001). Occupational therapists' perspectives of the needs of women in medium secure units. British Journal of Occupational Therapy, 64(9), 441448. http://doi.org/10.1177/030802260106400905

Bartlett, A., \& Somers, N. (2016). Are women really difficult? Challenges and solutions in the care of women in secure services. The Journal of Forensic Psychiatry \& Psychology, 28(2), 226-241. http://doi.org/10.1080/14789949.2016.1244281

Berg, A., \& Hallberg, I. (1999). Effects of systematic clinical supervision on psychiatric nurses' sense of coherence, creativity, work-related strain, job satisfaction and view of the effects from clinical supervision: A pre-post test design. Journal of Psychiatric and Mental Health Nursing, 6(5), 371-381. http://doi.org/10.1046/j.13652850.1999.00235.x

Beryl, R., Davies, J., \& Völlm, B. (2016). Lived experience of working with female patients in a high-secure mental health setting. International Journal of Forensic Mental Health Nursing. http://doi.org/10.1111/inm.12297

Bodner, E., Cohen-Fridel, S., Mashiah, M., Segal, M., Grinshpoon, A., Fischel, T., \& Iancu, I. (2015). The attitudes of psychiatric hospital staff toward hospitalization and treatment of patients with borderline personality disorder. BMC Psychiatry, 15, 1-12. http://doi.org/10.1186/s12888-014-0380-y

Braun, V., \& Clarke, V. (2006). Using thematic analysis in psychology. Qualitative Research in Psychology, 3(2), 77-101. 
Clark, A. (2006). Anonymising research data: Real life methods working paper. Retrieved from http://eprints.ncrm.ac.uk/480/

Compton-Dickinson, S., Odell-Miller, H., \& Adlam, J. (2012). Forensic music therapy: A treatment for men and women in secure hospital settings. London, UK: Jessica Kingsley Publishers.

Department of Health. (2002). Women's mental health: Into the mainstream - Strategic development of mental health care for women. London, UK: Department of Health.

Department of Health. (2003). Mainstreaming gender and women's mental health: Implementation guidance. London, UK: Department of Health.

De Vogel, V., \& Louppen, M. (2016). Measuring Feelings of Staff Members Towards Their Most Complex Female and Male Forensic Psychiatric Patients: A Pilot Study into Gender Differences. International Journal of Forensic Mental Health, 15(2), 174185. http://doi.org/10.1080/14999013.2016.1170741

Edge, D. (2005). Women's enhanced medium secure services (WEMSS): A scoping exercise for developing a research and evaluation strategy. London, UK: Department of Health.

Edge, D., Walker, T., Meacock, R., Wilson, H., McNair, L., Shaw, J., Gutridge, K., Mitchell, H., Robinson, L., Senior, J., Sutton, M., \& Abel, K. (2016). Secure pathways for women in the UK: Lessons from the Women's Enhanced Medium Secure Services (WEMSS) pilots. The Journal of Forensic Psychiatry \& Psychology, 1-20. http://doi.org/10.1080/14789949.2016.1244279

Elliott, K. A., \& Daley, D. (2013). Stress, coping, and psychological well-being among forensic health care professionals. Legal and Criminological Psychology, 18, 187204. http://doi.org/10.1111/j.2044-8333.2012.02045.x

Flick, U. (2009). An introduction to qualitative research: Edition 4. Los Angeles: Sage Publications. 
Grbich, C. (1999). Qualitative research in health: An introduction. London, UK: Sage Publications.

Grundy, A. C., Bee, P., Meade, O., Callaghan, P., Beatty, S., Olleveant, N., \& Lovell, K. (2015). Bringing meaning to user involvement in mental health care planning: A qualitative exploration of service user perspectives. Journal of Psychiatric and Mental Health Nursing, 23(1), 12-21. http://doi.org/10.1111/jpm.12275

Guest, G., MacQueen, K. M., \& Namey, E. E. (2012). Applied Thematic Analysis. Thousand Oaks, CA: Sage.

Howlett, M. (1994). Special hospitals service authority service for women patients. London, UK: SHSA.

James, D. V., Fineberg, N. A., Shah, A. K., \& Priest, R. G. (1990). An increase in violence on an acute psychiatric ward: A study of associated factors. British Journal of Psychiatry, 156, 846-852. http://doi.org/10.1192/bjp.156.6.846

Kurtz, A., \& Jeffcote, N. (2011). 'Everything contradicts in your mind': A qualitative study of experiences of forensic mental health staff in two contrasting services. Criminal Behaviour and Mental Health, 21(4), 245-258. http://doi.org/10.1002/cbm.796

Lincoln, Y.,\& Guba, E. (1985). Naturalistic Inquiry. Newbury Park, CA: Sage Publications.

Logan, C., \& Taylor, J. L. (2017) Working with personality disordered women in secure care: the challenge of gender-based service delivery. The Journal of Forensic Psychiatry \& Psychology, 28(2), 242-256. http://doi.org/10.1080/14789949.2017.1301531

Long, C. G., Harding, S., Payne, K., \& Collins, L. (2014). Nursing and health-care assistant experience of supervision in a medium secure psychiatric service for women: implications for service development. Journal of Psychiatric and Mental Health Nursing, 21(2), 154-162. http://doi.org/10.1111/jpm.12066 
May, N., \& Pope, C. (1995). Qualitative research: Rigor and qualitative research. British Medical Journal, 311, 109-112. http://doi.org/10.1136/bmj.311.6997.109

Mezey, G., Hassell, Y., \& Bartlett, A. (2005). Safety of women in mixed-sex and single-sex medium secure units: Staff and patient perceptions. British Journal of Psychiatry, 187,579-582. http://doi.org/10.1192/bjp.187.6.579

Moore, E. (2012). Personality disorder: its impact on staff and the role of supervision. Advances in Psychiatric Treatment, 18, 44-55. http://doi.org/10.1192/apt.bp.107.004754

NHS England. (2016). Women's enhanced medium secure services: Commentary on article. London, UK: NHS England.

Parry-Crooke, G., \& Stafford, P. (2009). My life: in safe hands? An evaluation of dedicated women's medium secure services in England. London: Department of Health.

Royal College of Psychiatrists. (2007). Standards for medium secure units. London, UK: Quality Network for Medium Secure Units.

Royal College of Psychiatrists. (2010). Your guide to relational security: See, Think, Act 2nd edition. Retrieved from https://www.rcpsych.ac.uk/pdf/STA_hndbk_2ndEd_Web_2.pdf

Shaw, J., Davies, J., \& Morey, H. (2001). An assessment of the security, dependency and treatment needs of all patients in secure services in a UK health region. The Journal of Forensic Psychiatry, 12(3), 610-637. http://doi.org/10.1080/09585180127380

Van Humbeeck, G., \& Van Audenhove, C. (2003). Expressed emotion of professionals towards mental health patients. Social Psychiatry and Psychiatric Epidemiology, 12, 232-235. http://doi.org/10.1017/s1121189x00003043

Walker, T., Logan, C., \& Shaw, J. (2017). Editorial: Women in Secure Care. The Journal of Forensic Psychiatry \& Psychology, 28(2). 
Yin, R. K. (2011). Qualitative Research from Start to Finish. New York, NY: The Guildford Press.

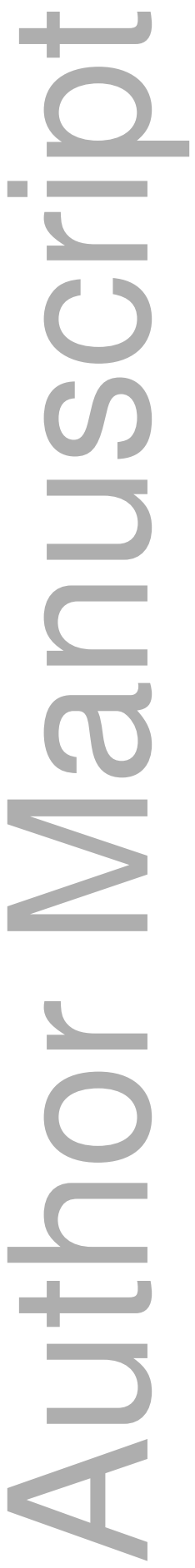




\section{Appendix 1: Interview Schedule}

\section{$\underline{\text { Introduction }}$}

- Role within the women's service

- History and how came to be working here

- Why work with women and how compares to working with men (WEMSS only)

- WEMSS vs non-WEMSS as staff

- What makes WEMSS enhanced

- WEMSS women vs non-WEMSS women

- How others view WEMSS service (non-WEMSS only)

- How view WEMSS service

\section{Staffing arrangements}

- General staffing levels \& roles

- Training and support - what is there, is it enough

- Opportunities for professional development

- Relationships with other staff

- Supervision

- Training to focus on women's needs and their ethnicity/culture/religion

- Effect of 1-1 observations

- $\quad$ Time spent escorting

- De-escalation techniques - comfortable/training

- Role of non-clinical staff

- Agency/bank staff

\section{Eligibility}

- Referral/assessment process

- Appropriateness of patient mix

- Patient's care pathway

\section{Service delivery}

- How service tailored specifically to women

- Views on self-harm, what support is offered 
- Strategies to prevent self-harm

- Offence related rehabilitation

- Degree of patient choice in care and treatment

- Ease of access to psychological therapies

- Patient views on psychological therapies

- Creative/complimentary therapies available

- What prevents delivering the ideal service

- How encourage women to engage

- Physical health care

- Women's centred services

- Nutrition/healthy diet

- How important do you think women's engagement with structured activities is in aiding their recovery?

- How many hours per week, on average, do you think each woman spends engaging in structured activity?

- Do you think that there are enough services and activities available?

- What do you think prevents the women on the ward from engaging in more services?

- Are there any strategies which you find help to encourage the women on your ward to engage in structured activities?

- Do you know how the current ward activity programme was designed?

\section{$\underline{\text { Service specification }}$}

- Impact of female-only patients on staff

- Advantages and disadvantages of gender-specific ward

\section{$\underline{\text { Relational security }}$}

- Understanding of relational security

- Training on relational security

- How maintain relational security

- What helps and hinders implementation

\section{Safety in the environment}

- Risk assessments - gender sensitive? 
- Main risks of group of women

- How risks and dangers assessed/addressed

- Security measures undertaken

- Do you feel safe?

\section{Built environment}

- Layout of building

- Improvements to physical ward environment

\section{$\underline{\text { Service standards }}$}

- Model/philosophy of care - description, how use on day-to-day basis

- Key performance indicators

\section{Care planning process and CPAs}

- Describe care planning process and who's involved

- How care plans are reviewed

- Do they take into account women's experience of previous trauma, for example violence or abuse

- How CPAs ensure ongoing care, safety and recovery

\section{Liaison and collaboration}

- Support for women interacting with community

\section{$\underline{\text { Levels of autonomy and control }}$}

- Understanding of autonomy

- How give women autonomy

\section{Discharge}

- Understanding of rehabilitation

- Process when approaching discharge, how involved are the women

- $\quad$ Support post-discharge?

\section{$\underline{\text { What works and frustrations }}$}


- Main factors in service working well

- Main difficulties and how cope

\section{Change and improvement}

- If asked to redesign service, what keep and what change

\section{$\underline{\text { Service provision }}$}

- Most important factor for women's recovery

- Does the service provide this

Is there anything else you think I should have asked/you'd like to tell me?
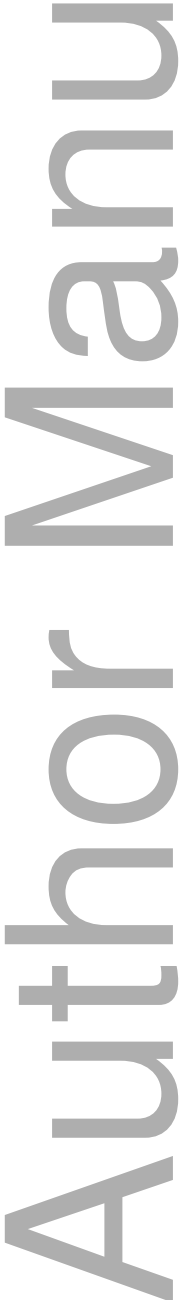

This article is protected by copyright. All rights reserved 
Table 1: Number of staff and occupations

\begin{tabular}{|l|c|c|}
\hline Role & WEMSS staff & Non-WEMSS staff \\
\hline $\begin{array}{l}\text { Nursing staff (ward based; including nursing } \\
\text { assistants and ward managers) }\end{array}$ & 3 & 4 \\
\hline Occupational therapy staff & 2 & 1 \\
\hline $\begin{array}{l}\text { Psychological therapies staff (including arts } \\
\text { therapies) }\end{array}$ & 1 & 1 \\
\hline $\begin{array}{l}\text { Consultant Psychiatrist/ Responsible } \\
\text { Clinician }\end{array}$ & 2 & 1 \\
\hline $\begin{array}{l}\text { Managerial staff (including business and } \\
\text { operational managers) }\end{array}$ & 1 & 2 \\
\hline
\end{tabular}




\section{University Library}

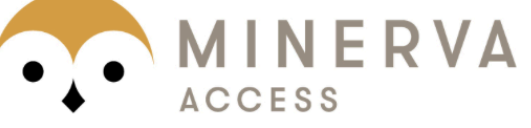

A gateway to Melbourne's research publications

Minerva Access is the Institutional Repository of The University of Melbourne

Author/s:

Walker, T;Edge, D;Shaw, J;Wilson, H;McNair, L;Mitchell, H;Gutridge, K;Senior, J;Sutton, M;Meacock, R;Abel, K

Title:

Contemporary women's secure psychiatric services in the United Kingdom: A qualitative analysis of staff views

Date:

2017-11-01

Citation:

Walker, T., Edge, D., Shaw, J., Wilson, H., McNair, L., Mitchell, H., Gutridge, K., Senior, J., Sutton, M., Meacock, R. \& Abel, K. (2017). Contemporary women's secure psychiatric services in the United Kingdom: A qualitative analysis of staff views. JOURNAL OF PSYCHIATRIC AND MENTAL HEALTH NURSING, 24 (9-10), pp.660-670. https:// doi.org/10.1111/jpm.12416.

Persistent Link:

http://hdl.handle.net/11343/293420 